autoimmunity. Autoimmunity. 2007;40(8):582-590.

11. Vang T, Miletic AV, Arimura Y, Tautz L, Rickert RC, Mustelin T. Protein tyrosine phosphatases in autoimmunity. Annu Rev Immunol. 2008;26:29-55.

12. Vang T, et al. Autoimmune-associated lymphoid tyrosine phosphatase is a gain-of-function variant. Nat Genet. 2005;37(12):1317-1319.

13. Zhang J, et al. The autoimmune disease-associated PTPN22 variant promotes calpain-mediated Lyp/Pep degradation associated with lymphocyte and dendritic cell hyperresponsiveness. Nat Genet. 2011;43(9):902-907.

14. Cambier JC, Gauld SB, Merrell KT, Vilen BJ. $\mathrm{B}$-cell anergy: from transgenic models to naturally occurring anergic B cells? Nat Rev Immunol. 2007;7(8):633-643

15. Pelanda R, Torres RM. Receptor editing for better or for worse. Curr Opin Immunol. 2006;18(2):184-190.

16. Pelanda R, Torres RM. Central B-cell tolerance: where selection begins. Cold Spring Harb Perspect
Biol. 2012;4(4):a007146.

17. Horikawa K, Nishizumi H, Umemori H, Aizawa S, Takatsu K, Yamamoto T. Distinctive roles of Fyn and Lyn in IgD- and IgM-mediated signaling. Int Immunol. 1999;11(9):1441-1449.

18. DeFranco AL, Chan VW, Lowell CA. Positive and negative roles of the tyrosine kinase Lyn in B cell function. Semin Immunol. 1998;10(4):299-307.

19. Castillejo-Lopez C, et al. Genetic and physical interaction of the B-cell systemic lupus erythematosus-associated genes BANK1 and BLK. Ann Rheum Dis. 2012;71(1):136-142.

20. Ravetch JV, Lanier LL. Immune inhibitory receptors. Science. 2000;290(5489):84-89.

21. Pao LI, et al. B cell-specific deletion of protein-tyrosine phosphatase Shp1 promotes B-1a cell development and causes systemic autoimmunity. Immunity. 2007;27(1):35-48.

22. O'Neill SK, et al. Monophosphorylation of CD79a and CD79b ITAM motifs initiates a SHIP-1 phosphatase- mediated inhibitory signaling cascade required for B cell anergy. Immunity. 2011;35(5):746-756.

23. Landego I, et al. Interaction of TAPP adapter proteins with phosphatidylinositol $(3,4)$-bisphosphate regulates B-cell activation and autoantibody production. Eur J Immunol. 2012;42(10):2760-2770.

24. Song MS, Salmena L, Pandolfi PP. The functions and regulation of the PTEN tumour suppressor. Nat Rev Mol Cell Biol. 2012;13(5):283-296.

25. Browne CD, Del Nagro CJ, Cato MH, Dengler HS, Rickert RC. Suppression of phosphatidylinositol 3,4,5-trisphosphate production is a key determinant of B cell anergy. Immunity. 2009;31(5):749-760.

26. Yokoyama K, et al. BANK regulates BCR-induced calcium mobilization by promoting tyrosine phosphorylation of IP(3) receptor. EMBOJ. 2002;21(1-2):83-92.

27. Fujimoto M, et al. CD19 regulates Src family protein tyrosine kinase activation in B lymphocytes through processive amplification. Immunity. 2000; 13(1):47-57.

\title{
Illuminating regeneration: noninvasive imaging of disease progression in muscular dystrophy
}

\author{
Jennifer R. Levy and Kevin P. Campbell \\ Howard Hughes Medical Institute, Department of Molecular Physiology and Biophysics, Department of Neurology, Department of Internal Medicine, \\ Roy J. and Lucille A. Carver College of Medicine, The University of lowa, lowa City, lowa, USA.
}

\begin{abstract}
Muscular dystrophies are characterized by progressive muscle weakness and wasting. Among the key obstacles to the development of therapies is the absence of an assay to monitor disease progression in live animals. In this issue of the JCI, Maguire and colleagues use noninvasive bioluminescence imaging to monitor luciferase activity in mice expressing an inducible luciferase reporter gene in satellite cells. These cells proliferate in response to degeneration, therefore increasing the level of luciferase expression in dystrophic muscle.
\end{abstract}

\section{Introduction}

Skeletal muscle has a robust regenerative capacity, with rapid reestablishment of full strength, even after severe damage to the tissue. Regeneration is mediated by muscle stem cells, called satellite cells. In response to muscle damage, satellite cells proliferate, differentiate into myoblasts, and fuse into myotubes, which act to repair damaged muscle. In muscular dystrophies, continuous muscle degeneration is accompanied by regeneration of muscle fibers mediated by satellite cell progeny (1).

Currently, the standard method for evaluating disease progression in muscular dystrophy animal models is muscle histopathology. This approach is labor inten-

Conflict of interest: The authors have declared that no conflict of interest exists.

Citation for this article: J Clin Invest. 2013; 123(5):1931-1934. doi:10.1172/JCI69568. sive, as it involves the removal and processing of the tissue of interest, imaging of the slides, and analysis of the images. Furthermore, the invasiveness of this approach does not permit consecutive sampling, hindering the ability to evaluate the course of a disease or success of a therapeutic strategy. Other methods for evaluating muscle disease include behavior testing and force testing of the dissected muscle, although the specificity of the results obtained from these tests can often be difficult to assess. High levels of serum biomarkers, such as serum creatine kinase, can be indicative of muscle damage, but levels depend on muscle mass and can be widely variable over time in individual dystrophic mice (2).

Perhaps the best candidate technology for studying muscle disease in live animals is MRI, which can reveal the permeability of muscle fibers correlating with disease severity (3). While MRI is noninvasive, it is more expensive and less widely available than bioluminescence imaging systems in animal research laboratories.

\section{A "regeneration reporter" mouse strain}

The first group to use bioluminescence imaging to reveal satellite cell proliferation was Sacco et al., who transplanted a single luciferase-expressing satellite cell into the tibialis anterior (TA) muscle of NOD/SCID mice that were depleted of endogenous satellite cells by irradiation (4). They observed that a single luciferase-expressing satellite cell is capable of self renewal after transplantation. Further, they found a substantial increase in satellite cell proliferation, as indicated by increased bioluminescence values, in response to muscle tissue damage by notexin.

In this issue, Maguire et al. (5) utilized the Pax7Cre ${ }^{E R} /$ LuSEAP mouse first generated by Nishijo et al. (6) to develop a mouse model that could be used to monitor muscle regeneration in response to disease and injury. This mouse expresses a Cre-dependent firefly luciferase gene and an estrogen-responsive Cre-recombinase under the control of the Pax7 locus. Because satellite cells are the only muscle cells in the adult that express Pax7, these mice 


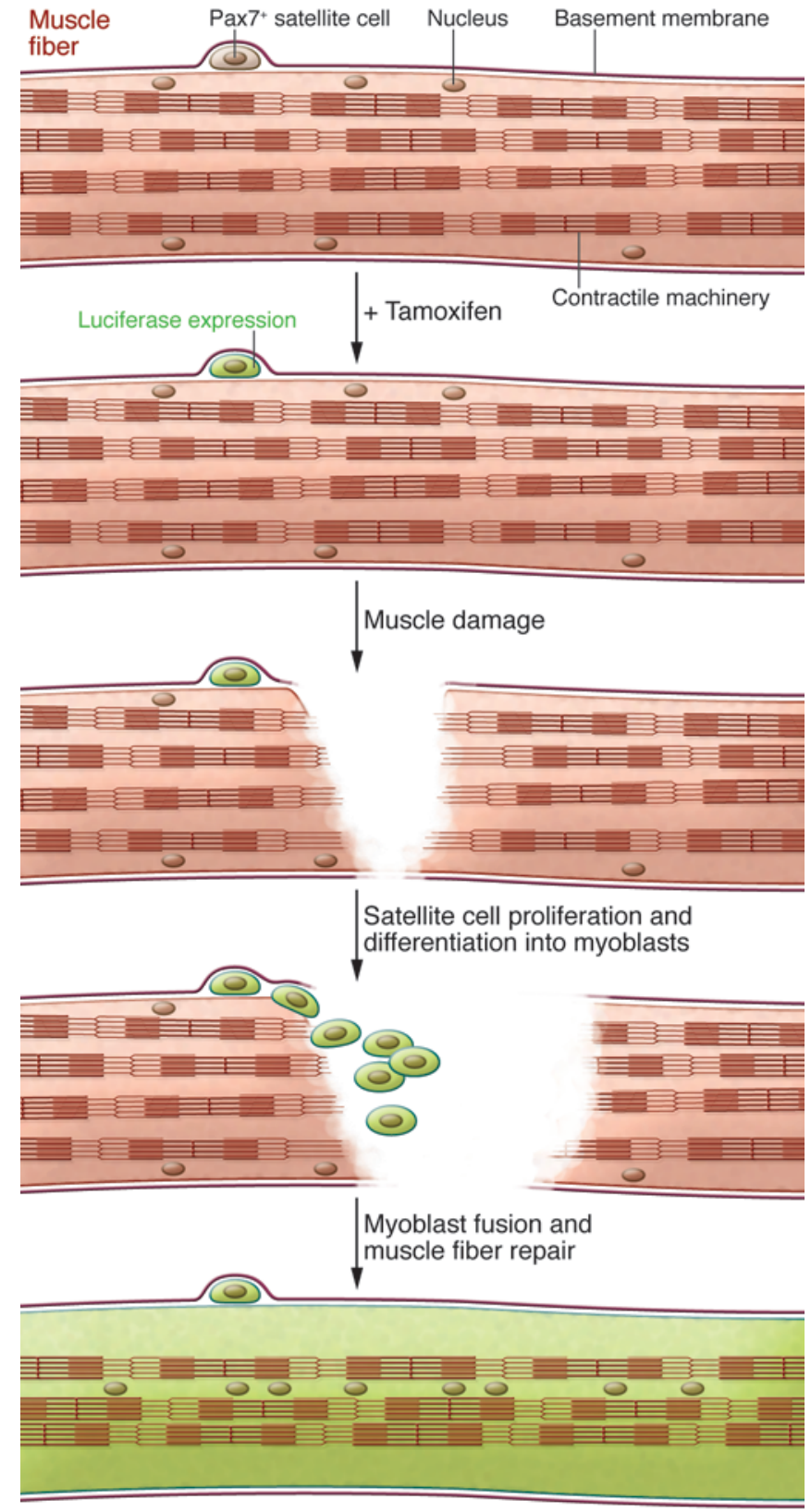

express the bioreporter following tamoxifen treatment specifically in satellite cells. This expression is maintained as satellite cell progeny proliferate and differentiate into myotubes to repair the muscle, and expression of luciferase can be monitored by noninvasive, bioluminescence imaging (Figure 1). Nishijo et al. previously utilized the Pax7Cre $e^{E R} / L u S E A P$ mice to characterize satellite cell kinetics in growing postnatal muscle and found a doubling of the signal every 3.93 weeks from adolescence to young adulthood (6). Maguire et al. use the Pax7Cre ER/LuSEAP mouse to assess satellite cell activation and proliferation as an indicator of regeneration. They first injured the TA muscles with cardiotoxin and compared the luciferase signal between the injured and uninjured limbs. Luciferase activity increased following injury, and immunohistochemical studies confirmed that luciferase-expressing cells were present in the injured TA as nascent myofibers and myotubes (5). This suggests that the noninvasive measurement of luciferase activity in the Pax7Cre $e^{E R} / L u S E A P$ strain is an accurate measure of the proliferative activity of satellite cells and therefore has potential to be used to measure muscle regeneration in muscular dystrophy models as well.

\section{Figure 1}

A "regeneration reporter" mouse strain. Pax7-positive muscle satellite cells express luciferase (indicated by green shading) following tamoxifen injection. Upon damage to muscle by either cardiotoxin injury or disease, satellite cells differentiate, and the progeny also express luciferase. Satellite cell progeny proliferate and differentiate into myotubes to repair the muscle, increasing the luciferase signal that can be detected by a bioluminescence imager.

\section{Using luciferase to monitor disease progression}

A key feature of dystrophic muscle is the degeneration of mature muscle fibers and the subsequent fusion of satellite cellderived myoblasts to form myotubes, which mature into fibers. This degeneration/ regeneration is apparent by muscle histopathology, where regenerating fibers can be identified by their centrally located nuclei. Maguire and colleagues hypothesized that

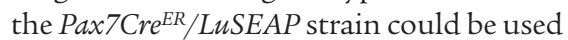
to develop a noninvasive and quantifiable measure of regeneration activity in dystrophic mice. To test this, they crossed this 
reporter strain with a dysferlin-deficient $\left(\right.$ Dysf $\left.{ }^{-}\right)$mouse model of limb girdle muscular dystrophy 2B (LGMD2B) $(7,8)$. Dysferlin is a transmembrane protein involved in calcium-mediated plasma membrane repair (9). Like human LGMD2B patients, Dysf $f^{\prime-}$ mice develop a slowly progressive muscular dystrophy, mainly in proximal limb muscles. Muscles from Dysf ${ }^{-1}$ mice exhibit centrally nucleated fibers, necrotic fibers, fat deposition, and inflammatory cell infiltrates (10).

The authors injected 2-month-old Dysf /-) Pax7Cre ER $_{\text {LuSEAP mice with tamoxifen to }}$ induce luciferase expression in satellite cells and found luciferase expression increased over time in the hind limb muscles of dysferlin-deficient, but not wild-type, mice. They found luciferase activity mainly in the proximal limb muscles as early as 3 months of age, with some involvement in the distal muscles starting at 6 months, and increasing luciferase activity in both proximal and distal muscle groups up to 18 months of age. Further examination of these mice showed a correlation between intensity of luciferase signal (as determined by bioluminescent imaging), number of luciferase positive fibers (as determined by immunohistochemistry), and extent of histopathology, including centrally nucleated fibers and fibers that express embryonic myosin heavy chain, a marker for newly regenerated myofibers (5). This validates luciferase imaging as an alternative to conventional measures of disease progression in muscular dystrophy.

There are several immediate advantages to using bioluminescent imaging to determine the extent of disease in muscular dystrophy models. First, the method is less labor intensive and more quantitative than classic histopathology. Second, measurements for individual mice are consistent and show patterns of luciferase activity that parallel the entire cohort. This suggests that a bioluminescence scheme for quantifying regeneration activity could be useful in therapeutic studies, in which each mouse could be used as its own pre- and posttreatment control. A third advantage to this system is the apparent increased sensitivity compared with conventional measures. The Dysf/- mouse has been previously characterized as having a slowly progressive muscular dystrophy by 6 months of age, followed by rapid disease progression $(7,8)$. This is similar to other dysferlin-deficient LGMD2B mouse models (the naturally occurring A/J mice (11) and a targeted dysferlin knockout (12) both show active myopathy at approximately 6 to 8 months). Excitingly, the current paper finds a significant increase in luciferase expression in Dysf $f^{-}$mice as early as 3 months. This suggests that researchers may now have the opportunity to study the early pathophysiology of dysferlin deficiency and test the effectiveness of early therapeutic interventions.

One major limit to a bioluminescencebased reporter of regeneration activity is that it is light based. Therefore, luciferase signal is influenced by hair, skin pigmentation, thickness of skin and fat, and depth of tissue. This is particularly unfortunate in the case of the diaphragm, which is severely affected in several muscular dystrophies (especially Duchenne, ref. 13). Due to the depth of the diaphragm and the amount of tissue through which light must travel to image it, this method would not be particularly conducive to evaluating regeneration in the diaphragm. For researchers interested in regeneration activity in limb muscles, however, a bioluminescence strategy appears to have potential as a reliable and sensitive technique. Another disadvantage to this technique is the limited potential for an equivalent diagnostic tool for human patients. While therapeutic studies can be designed on animal models using luciferase activity as an indicator of regeneration activity, subsequent testing in humans will need to utilize a different technique, such as MRI imaging, to measure outcome. Finally, regeneration, while closely linked, may not be directly proportional to disease severity. Several mouse models have been generated that have regenerative defects (for examples, see ref. 14). Breeding these mice with the Pax $7 C r e^{E R} / L u S E A P$ reporter line may give an incomplete picture of disease progression. Researchers must keep this caveat in mind when utilizing this technique on mouse models where the satellite cell regenerative capacity has not yet been characterized.

\section{Future applications}

Maguire and colleagues have demonstrated that bioluminescence imaging of satellite cells is an accurate representation of regeneration activity in a mouse model of LGMD2B. By crossing the Pax7Cre ER/ LUSEAP mouse to other muscular dystrophy models (the $m d x$ model of Duchenne, for example), this strategy can easily be used to study muscle degeneration and regeneration in any number of muscle diseases. Further, the Pax7Cre ER/LuSEAP mouse can be used to examine satellite cell proliferation in models of atrophy and hypertrophy (hind limb suspension and external loading, respectively), as well as other neuromuscular disorders. In the case of LGMD2B, the authors found that luciferase activity was significantly increased compared with wild-type controls at ages as early as 3 months. Bioluminescence imaging may also reveal early time points at which a difference can be detected in other models of muscle disease, which could provide insights into disease mechanism and alter the timing in which researchers consider applying therapies.

The Pax7Cre ER/LuSEAP mouse expresses luciferase in differentiated satellite cells, regardless of fate (myogenic, adipogenic, or fibrogenic). In the future, it would be interesting to utilize key transcription factors in order to design a reporter that could differentiate between self-renewed satellite cells and differentiated satellite cell progeny.

Compared to classical histology, which requires substantial effort in the dissection, processing, imaging, and analysis of each tissue, bioluminescence imaging is high throughput and quantitative. In addition, it is noninvasive and therefore it is ideal for studies in which each animal, and even each muscle, can be used as its own control. This technology is certainly an avenue investigators should consider when designing future studies of regeneration activity and therapeutic intervention in muscular disease.

\section{Acknowledgments}

J.R. Levy is supported by a Muscular Dystrophy Association Development grant (MDA200826). K.P. Campbell is an investigator of the Howard Hughes Medical Institute.

Address correspondence to: Kevin P. Campbell, Howard Hughes Medical Institute, Department of Molecular Biology and Biophysics, The University of Iowa College of Medicine, 4283 CBRB, 285 Newton Road, Iowa City, Iowa, 52242-1101, USA. Phone: 319.335.7867; Fax: 319.335.6957; E-mail: kevin-campbell@uiowa.edu.

1. Yin H, Price F, Rudnicki MA. Satellite cells and the muscle stem cell niche. Physiol Rev. 2013;93(1):23-67.

2. Lieberman JS, Taylor RG, Fowler WM. Serum creatine phosphokinase variations in dystrophic mice. Exp Neurol. 1981;73(3):716-724.

3. McIntosh L, Granberg KE, Briere KM, Anderson JE. Nuclear magnetic resonance spectroscopy study of muscle growth, mdx dystrophy and glucocorticoid 
treatments: correlation with repair. NMR Biomed. 1998;11(1):1-10.

4. Sacco A, Doyonnas R, Kraft P, Vitorovic S, Blau HM. Self-renewal and expansion of single transplanted muscle stem cells. Nature. 2008; 456(7221):502-506.

5. Maguire KK, Lim L, Speedy S, Rando TA Assessment of disease activity in muscular dystrophies by noninvasive imaging. $J$ Clin Invest. 2013;123(5):2298-2305.

6. Nishijo K, et al. Biomarker system for studying muscle, stem cells, and cancer in vivo. FASEB J. 2009;23(8):2681-2690.
7. Bittner RE, et al. Dysferlin deletion in SJL mice (SJLDysf) defines a natural model for limb girdle muscular dystrophy 2B. Nat Genet. 1999;23(2):141-142.

8. Weller AH, Magliato SA, Bell KP, Rosenberg NL. Spontaneous myopathy in the SJL/J mouse: pathology and strength loss. Muscle Nerve. 1997; 20(1):72-82.

9. Han R, Campbell KP. Dysferlin and muscle membrane repair. Curr Opin Cell Biol. 2007;19(4):409-416.

10. Hornsey MA, Laval SH, Barresi R, Lochmüller $\mathrm{H}$, Bushby K. Muscular dystrophy in dysferlin-deficient mouse models. Neuromuscul Disord. 2013;23(5):377-387.
11. Ho M, et al. Disruption of muscle membrane and phenotype divergence in two novel mouse models of dysferlin deficiency. Hum Mol Genet. 2004;13(18):1999-2010.

12. Bansal D, et al. Defective membrane repair in dysferlin-deficient muscular dystrophy. Nature. 2003;423(6936):168-172.

13. Stedman HH, et al. The mdx mouse diaphragm reproduces the degenerative changes of Duchenne muscular dystrophy. Nature. 1991;352(6335):536-539.

14. Wallace GQ, McNally EM. Mechanisms of muscle degeneration, regeneration, and repair in the muscular dystrophies. Annu Rev Physiol. 2009;71:37-57.

\title{
Where hypertension happens
}

\author{
Timothy L. Reudelhuber
}

\begin{abstract}
Laboratory of Molecular Biochemistry of Hypertension, Clinical Research Institute of Montreal, Division of Experimental Medicine, Department of Medicine, McGill University, Montreal, Quebec, Canada. Department of Medicine, University of Montreal, Montreal, Quebec, Canada.
\end{abstract}

\begin{abstract}
Essential hypertension, which accounts for $\mathbf{9 0 \%}-\mathbf{9 5 \%}$ of all cases of hypertension seen in the clinic, is also referred to as idiopathic hypertension, because we simply don't understand the cause(s). Although many theories have been advanced, in the current issue of the JCI, Gonzalez-Villalobos et al. present further evidence implicating the intrarenal renin-angiotensin system and take us one step further by proposing a mechanism underlying this pathology.
\end{abstract}

We know a lot about the environmental triggers for essential hypertension; there's no doubt that aging, obesity associated with increased insulin resistance, excessive alcohol intake, ethnicity, stress, and (in certain individuals) excessive dietary salt intake or inadequate dietary potassium or calcium can contribute to high blood pressure (1). Nevertheless, we don't have a clear picture of what happens in the body to translate most of these stimuli into a pathological condition. Any attendee of a recent hypertension research meeting will know that there is no shortage of hypotheses, including increased production of reactive oxygen species, enhanced reactivity of resistance blood vessels to vasoconstriction, vascular inflammation involving immune cells, and malfunctioning baroreceptors or triggers in the central nervous system. Although animal models exist in which each of these mechanisms can be shown to alter blood pressure and each may indeed contribute to a greater or lesser degree to elevating blood pres-

Conflict of interest: The author has declared that no conflict of interest exists.

Citation for this article: J Clin Invest. 2013; 123(5):1934-1936. doi:10.1172/JCI69296. sure in hypertensive patients, many lines of evidence suggest that it's in the kidney that it all comes together.

\section{The kidney as a critical hypertension locus}

Several researchers have demonstrated that transplanting the kidneys of hypertensive rats into normotensive control rats rendered the recipients hypertensive (2-4). A molecular-era refinement of such transplant experiments suggests that the hypertension that travels with the kidney might be due in part to the renin-angiotensin system (RAS). In the classical RAS, angiotensinogen from the liver is cleaved by kidney-derived renin to form angiotensin I, which is subsequently cleaved by angiotensinconverting enzyme (ACE) present on the surface of endothelial cells throughout the body to generate the potent vasopeptide angiotensin II (Figure 1A). There are two angiotensin II receptors, and it is the angiotensin II receptor type $1\left(\mathrm{AT}_{\mathrm{R}}\right)$ subtype that increases blood pressure and fluid volume through vasoconstriction and stimulation of aldosterone secretion from the adrenal gland and salt retention by the kidneys. Coffman and colleagues transplanted the kidneys of $A T 1_{R}$-defi- cient mice into control animals and vice versa (5). Subsequent infusion of these mice for two weeks with a high dose of angiotensin II revealed that the mice with AT $1_{R}$ expression in the kidney, but a complete lack of this receptor elsewhere in the body, developed hypertension and cardiac hypertrophy. Surprisingly, the mice that lacked $\mathrm{AT} 1_{\mathrm{R}}$ in the kidney developed neither hypertension nor cardiac hypertrophy, despite expressing AT $1_{\mathrm{R}}$ everywhere else in the body. The overall implication of these studies is that angiotensin II sensing within the kidney is a critical mediator of hypertension.

However, a model in which circulating angiotensin II causes hypertension by a direct action on the kidney fails to explain a long-standing clinical conundrum: how does ACE inhibition remain effective in lowering blood pressure in individuals whose circulating angiotensin II gradually returns to pretreatment levels (socalled angiotensin escape) (6)? In this issue of the JCI, Gonzalez-Villalobos et al. address this question by investigating the role of the intrarenal RAS in hypertension by inactivating the Ace gene in mouse kidney (7). The way that they achieved this was somewhat unconventional. They began with mice that had a whole-body inactivation of Ace. However, because the complete inactivation of any of the RAS genes, including Ace, results in severe renal defects and high neonatal lethality in both humans and mice (8), Gonzalez-Villalobos et al. generated Ace knockout mice that ectopically expressed ACE in either hepatocytes (ACE $3 / 3$ mice) 